\title{
Increased Laterality of the Thalamus in Children and Adolescents with Asperger's Disorder: An MRI and Proton Spectroscopy Study
}

\author{
Gökçe Nur Say ${ }^{\circledR}$, Bünyamin Şahin², Kerim Aslan³, Seher Akbaş ${ }^{1}$, Lütfi İncesu ${ }^{3}$, and Meltem Ceyhan ${ }^{3}$ \\ ${ }^{1}$ Department of Child and Adolescent Psychiatry, Faculty of Medicine, Ondokuz Mayıs University, Samsun, Turkey \\ ${ }^{2}$ Department of Anatomy, Faculty of Medicine, Ondokuz Mayıs University, Samsun, Turkey \\ ${ }^{3}$ Department of Radiology, Faculty of Medicine, Ondokuz Mayıs University, Samsun, Turkey
}

\begin{abstract}
Objective Thalamic abnormalities have been reported in people with pervasive developmental disorders (PDD) including Asperger's Disorder (ASP). The aim of the present study was to compare the volume and volume fraction of the thalamus and the metabolite concentrations in children and adolescents with ASP using the magnetic resonance imaging and proton magnetic resonance spectroscopy. Additionally, the relationships between thalamic abnormalities and clinical features were examined.
\end{abstract}

Methods Volume and volume fractional and metabolic measurements of bilateral thalamus were collected from 15 boys with ASP with a total IQ over 70 (age range 7-18 years, mean age 11.6 \pm 3.79 years), and 15 healthy controls matching age, sex and IQ. The thalamic volumes, hemisphere volumes and total brain volumes (TBV) were estimated using the stereological methods on magnetic resonance images. Chemical metabolites of thalamus were evaluated by ${ }^{1} \mathrm{H}$ spectroscopy.

Results No differences in thalamic volumes, volume fractions and metabolites were observed between the groups. There were significant correlation between thalamic volume and total brain volume in both groups. The ASP group showed a significant left-minus-right thalamus difference as well as a significantly greater laterality index. In addition, a significant correlation between the laterality index and Autism Behavior Checklist language scores was observed.

Conclusion Findings from this investigation point to a significant increase in laterality of the thalamus and a relationship with language problems in individuals with ASP. Our findings suggest that thalamic abnormalities may be related to mild language problems observed in ASP.

Psychiatry Investig 2014;11(3):237-242

Key Words Asperger, Autism, Thalamus, Laterality, Language, Children.

\section{INTRODUCTION}

Asperger's Disorder (ASP) is a pervasive developmental disorder (PDD) characterized by abnormalities in reciprocal social interactions, as well as restricted, repetitive patterns of behavior, interests and activity. The basic difference between ASP and autistic disorder is that in ASP, there is no evident delay and impairment in language and cognitive development. ${ }^{1}$

The thalamus is involved in most physiological processes and is affected by many neurologic and psychiatric disorders.

Received: May 10, 2013 Revised: July 29, 2013

Accepted: August 3, 2013 Available online: July 21, 2014

$\bowtie$ Correspondence: Gökçe Nur Say, MD

Ondokuz Mayıs Üniversitesi Tip Fakültesi Hastanesi, Cocuk ve Ergen Psikiyatrisi Anabilim Dal1, Kurupelit, Samsun 55139, Turkey

Tel: +90 50567141 92, Fax: +903624576041

E-mail: gokcenurtasdemir@yahoo.com.tr

(a) This is an Open Access article distributed under the terms of the Creative Commons Attribution Non-Commercial License (http://creativecommons.org/licenses/by$\mathrm{nc} / 3.0$ ) which permits unrestricted non-commercial use, distribution, and reproduction in any medium, provided the original work is properly cited.
The thalamus functions in the integration of sensory input, cortical arousal, memory and language. ${ }^{2}$

Evidence of thalamic abnormalities has been accumulating in people with PDD including ASP. However, there are few studies concerning the role of the thalamus in pediatric age autistic people. Additionally, little is known about the link between neurobiologic abnormalities and clinical features of autism.

Previous studies evaluating the volume and volume fraction of the thalamus in autism using magnetic resonance imaging (MRI), no significant differences were determined between the patient groups and the healthy controls. ${ }^{3-5}$ However, the fraction of thalamic volume to total brain volume (TBV) was found to be abnormal both in the high functioning autism (HFA) $)^{3,4}$ and ASP cases. ${ }^{5}$ In a study involving only children and adolescents, a correlation between thalamic volume and total brain volume was observed in the autism group like the healthy controls. ${ }^{6}$ In a recent study, reduced thalamic volume was ob- 
served in all autism subgroups including ASP. ${ }^{7}$

There are few proton magnetic resonance spectroscopy $\left({ }^{1} \mathrm{H}\right.$ MRS) studies examining the thalamus. In a study by Perich et al., ${ }^{8} \mathrm{~N}$-Acetylaspartate (NAA)/creatine (Cre), as a marker of functional neuroaxonal tissue, was found to be low in the autism group aged 8-13 years. Another study reported significantly reduced NAA level in the left thalamus of children and adolescents with HFA. ${ }^{6}$

The main hypothesis of this study was the adolescents with ASP may have structural and chemical abnormalities. We aimed to evaluate volumetric and volume fractional differences of the thalamus and cerebral cortices in boys with ASP using stereological techniques on MR images. Additionally, the chemical metabolites of the thalamus [NAA, Choline (Cho), $\mathrm{Cre}]$ were evaluated by ${ }^{1} \mathrm{H}-\mathrm{MRS}$. We also examined the relationships between volumetric and metabolic abnormalities and clinical features.

\section{METHODS}

\section{Participants}

We compared the findings of 15 right-handed boys with ASP with 15 healthy right-handed boys' data. The mean ages of the ASP and control groups were matching and ranging between $7-18$ years old.

Individuals with ASP were recruited from outpatients of the Department of Child and Adolescent Psychiatry, Ondokuz Mayis University Medical Hospital, Samsun, Turkey. Inclusion in the ASP group was contingent on the following criteria: 1) a diagnosis of ASP according to DSM-IV criteria, 2) male gender, 3) chronological age between 7-18 years, 4) right handedness, 5) verbal, performance and full scale IQ $>70$. Exclusion criteria for the ASP group were the presence of any neurological, metabolic, infectious or genetic disorder, history of seizure, head trauma or asphyxia. The diagnosis of ASP was made using the strict DSM-IV criteria. Diagnoses were based on the information from interviews with the parent and child and clinical examination. The patients were assessed by two expert child and adolescent psychiatrists individually. Patients were included if there was a consensus for the diagnosis of ASP by two clinicians.

The control group consisted of healthy volunteers recruited from the community. Right-handed male subjects who were the same age as the ASP subjects were selected.

Exclusion criteria for the control group included any present or lifetime mental or medical disorder, history of asphyxia, head trauma or seizure. Control subjects with a verbal, performance or full scale IQ $<70$ or subjects with a family history of neuropsychiatric disorders such as PDD, schizophrenia, affective disorder or mental motor retardation were also excluded.

\section{Instruments}

Symptom severity of individuals with ASP was assessed with the Autism Behavior Checklist $(\mathrm{ABC})$ which was rated by the parents. The $\mathrm{ABC}$ consists of 57 items, and 5 categories: Sensory, Relating, Body and Object Use, Language, Social and Self Help. For the Turkish version of the ABC total score Cronbach's alpha reliability coefficient was 0.96 and SpearmanBrown two half-split coefficient was 0.96 . Findings suggested that the validity and the reliability of $A B C$ were satisfactory for the Turkish sample. ${ }^{9}$

All control subjects were assessed with the Schedule for Affective Disorders and Schizophrenia for School Age ChildrenPresent and Lifetime Version ${ }^{10}$ (KSADS-PL). The reliability and validity study was conducted for the Turkish version of KSADS-PL. ${ }^{11}$

Standardized cognitive testing with the Weschler Intelligence Scale for Children, Revised (WISC-R) or the Weschler Adult Intelligent Scale, Revised (WAIS-R) was administered to all participants. The dominant hand side of all participants was assessed according to the information obtained from participants and their parents.

\section{Ethics}

Written informed consent was obtained from the parents of all subjects. The research was approved by the Ethics Committee of the Faculty of Medicine, Ondokuz Mayis University.

\section{MRI scans and structural measurements}

The MR and H-MRS images were obtained in the same session on a 1.5T scanner (Magnetom, Symphony-Quantum, Siemens Medical Systems, Erlangen, Germany) using a standard head coil.

Structural imaging sequences included a 3D, T1-weighted spoiled gradient recalled (SPGR) acquisition, with echo time $=5.2 \mathrm{~ms}$ and repetition time $=11 \mathrm{~ms}$ (NEX 1, flip angle, $15^{\circ}$ ). The sequence involved the acquisition of 176 contiguous coronal slices with a slice thickness of $1 \mathrm{~mm}$, field of view of $256 \mathrm{~mm}$, and a matrix of $224 \times 256$.

The images were stored as the DICOM files in CDs. The DICOM images were exported to JPEG files for the stereological analysis. The images transferred to the ImageJ which is a free software distributed by the National Institute of Health, the United States of America. The volume and volume fraction of the thalamus and the volume of the total brain were estimated using the ImageJ. For the volume estimation of the thalamus the images opened in the software and 1 out of 2 images were sampled to proceed depending on the rules of the systematic random sampling. For the hemispheres, 1 out of 10 images were sampled.

Coronal plane images with $1 \mathrm{~mm}$ thickness were used both 
for the estimation of the thalamus and the cerebral hemispheres. The boundaries of the thalamus were delineated from the rostral end to the caudal end. Manual planimetry was used for this purpose and the software calculated the sectional surface area of the thalamus in the examined section automatically (Figure 1). For the delineation of the cerebral hemispheres the sampled image series were used. The borders of the hemisphere were drawn to split them from the opposite site hemisphere, cerebellum and brainstem. Semi-automated planimetry was used for the assessment of the sectional surface area of the hemisphere. For this purpose, the threshold function of the program was used. The ROI of the section were labeled and selected. The software measured the sectional surface area of the hemisphere automatically. The same procedure was done for both hemispheres separately.

The morphometric analysis was done by one observer who was blind for the groups and the patients.

Obtained sectional surface areas were used for the estimations of volumes. The thalamic volumes, hemisphere volumes and total brain volumes (TBV) were estimated using the Cavalieri principle of the stereological methods as described in previous studies. ${ }^{12,13}$ For the estimation of the volumes the sum of the obtained sectional surface areas was multiplied by the section thickness of the images $(2 \mathrm{~mm}$ for the thalamus and 10 $\mathrm{mm}$ for the hemisphere). Dividing the volume of thalamus to the volume of hemisphere and multiplying the data with 100 assessed the volume fraction of the thalamus within the hemisphere in percentages. The volume data were also used for the assessment of the laterality index. The laterality index was calculated using the following formula: [(Left-Right)/[(Left+ Right) $] \times 100$. The laterality is measured by the absolute value of left-minus-right thalamus difference. All calculations and other related data were obtained as a spread sheet using the Microsoft Excel. After initial setup and preparation of the formulae, the cross-sectional surface areas and formulae were entered for each subject and the final data were obtained automatically.

\section{${ }^{1} \mathrm{H}$ spectroscopy}

Before performing H-MRS, T2 weighted images in coronal, axial and sagittal planes of the whole brain (section thickness, $5 \mathrm{~mm}$, FOV, $230 \mathrm{~cm}$; TR $4270 \mathrm{~ms}$; TE, $108 \mathrm{~ms}$; flip angle, 150; matrix, $199 \times 384$ ) were acquired to define the region of interest (ROI). A 2D, multivoxel H-MRS was performed using chemical shift imaging (CSI) with the scanning parameters of TR 1500, TE 135, NEX4, spectral width $1000 \mathrm{~Hz}$, FOV 4.

The region of interest (ROI) encompassed the right and left thalamus with right-left and anterior-posterior dimensions of $50 \times 60 \mathrm{~mm}$. MR spectra were acquired from a $1 \mathrm{~mL}$ $\left(10 \times 10 \times 10 \mathrm{~mm}^{3}\right)$ volume prescribed over right and left thal- amus separately (Figure 2). The H-MRS data were analyzed using the current workstation (Leonardo, Siemens Medical Solutions, Forcheim, Germany). Quantitative analysis of spectra

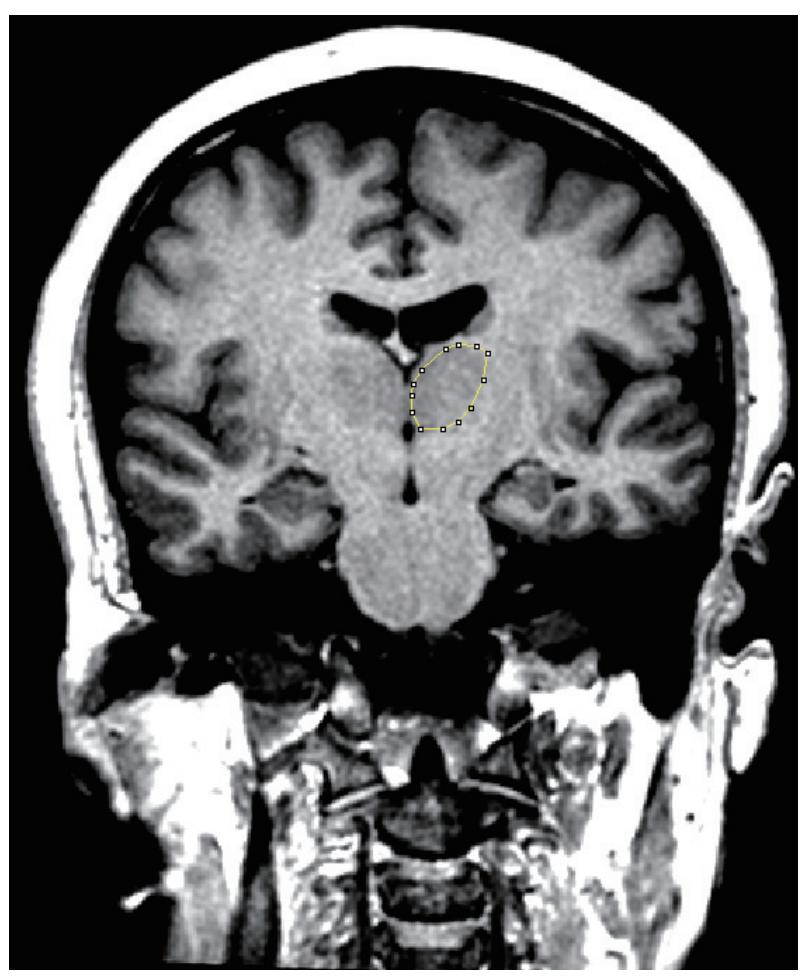

Figure 1. Boundaries of thalamus used for volumetric estimation on coronal plane.

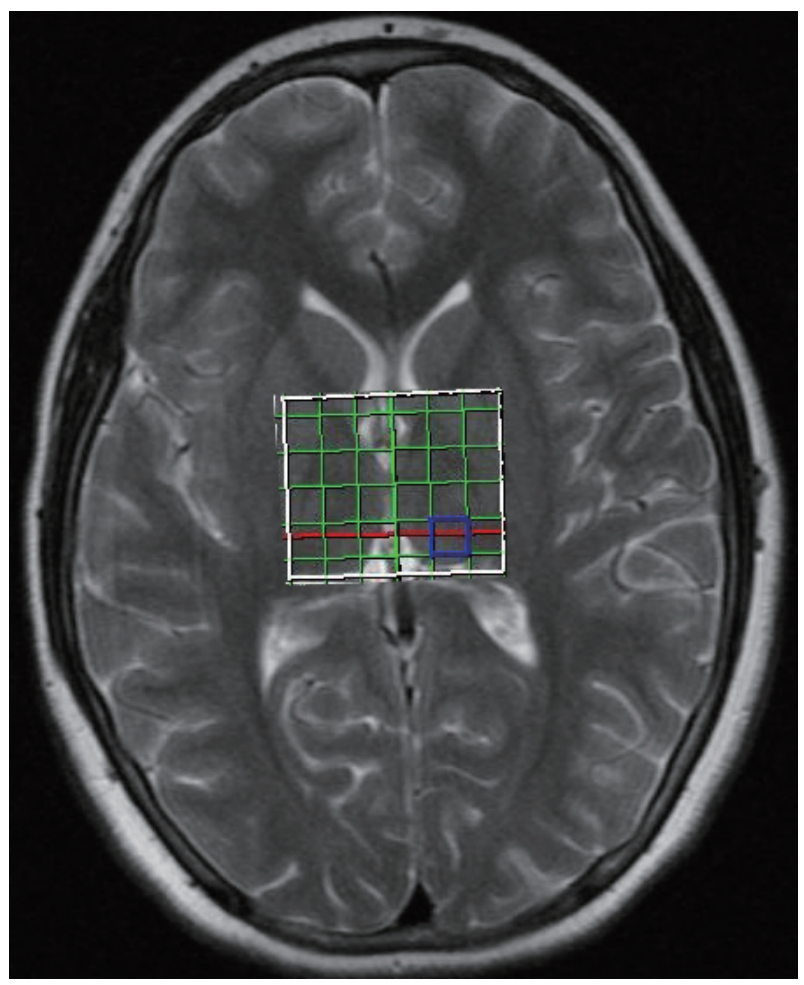

Figure 2. Thalamic voxel placement. 
was confined to NAA (chemical shift $2.02 \mathrm{ppm}$ ), Cre (3.02 $\mathrm{ppm})$, Cho (3.22 ppm). Concentrations of metabolites were determined using areas under the spectroscopic peaks.

\section{Data analysis}

Data were analyzed using the SPSS for Windows version 16 (SPSS Inc., Chicago, IL, USA) and reported as mean and standard deviation $(\mathrm{M} \pm \mathrm{SD})$. The metabolite concentrations, the thalamus volumes and IQ scores were found to be normally distributed in both groups according to the Shapiro-Wilks test. Parametric tests (two-tailed Student t-test) were used to analyse normally distributed data. Nonparametric tests (MannWhitney $\mathrm{U}$ test) were used for the data that did not follow a

Table 1. Demographic information

\begin{tabular}{|c|c|c|c|c|}
\hline & \multirow{3}{*}{$\begin{array}{c}\text { ASP } \\
\mathrm{N}=15 \\
\text { Mean } \pm S D\end{array}$} & \multirow{3}{*}{$\begin{array}{c}\text { Control } \\
\mathrm{N}=15 \\
\text { Mean } \pm \mathrm{SD}\end{array}$} & \multirow{2}{*}{\multicolumn{2}{|c|}{$\begin{array}{l}\text { t-test } \\
\mathrm{df}=28\end{array}$}} \\
\hline & & & & \\
\hline & & & $\mathrm{t}$ & $\mathrm{p}$ \\
\hline Age & $11.6 \pm 3.79$ & $11.6 \pm 3.79$ & - & - \\
\hline Verbal IQ & $102.87 \pm 14.99$ & $101.93 \pm 7.57$ & 0.215 & 0.832 \\
\hline Performance IQ & $99.93 \pm 16.45$ & $99.00 \pm 10.03$ & 0.188 & 0.853 \\
\hline Full-scale IQ & $102.33 \pm 15.90$ & $100.60 \pm 9.45$ & 0.363 & 0.720 \\
\hline $\mathrm{ABC}$ & & & & \\
\hline Sensory & $1.26 \pm 1.62$ & - & - & - \\
\hline Relating & $6.33 \pm 5.16$ & - & - & - \\
\hline Body \& object use & $2.33 \pm 3.37$ & - & - & - \\
\hline Language & $3.73 \pm 3.73$ & - & - & - \\
\hline Social \& Self Help & $4.40 \pm 2.79$ & - & - & - \\
\hline Total & $18.06 \pm 10.18$ & - & - & - \\
\hline
\end{tabular}

SD: standard deviation, ABC: Autism Behavior Checklist, ASP: Asperger's disorder group, IQ: intelligence quotient normal distribution (hemisphere volumes and TBV). Spearman's correlation coefficients were used to examine the association between thalamic volumes and TBV. The association between the laterality index and $\mathrm{ABC}$ scores was also analyzed using the Spearman's correlation coefficients. Analysis of covariance (ANCOVA) was used to compare the thalamus volumes of the two groups after controlling for the confounding effect of the TBV. A value of $\mathrm{p}<0.05$ was accepted as statistically significant.

\section{RESULTS}

The age ranges and the mean ages of the groups were identical since the ASP and control subjects were matched one to one in terms of age ( $7-18$ years, mean $=11.6$ years, $S D=3.79$ years). No significant differences were found between individuals with ASP and controls in verbal, performance and full IQ scores. Table 1 summarizes the mean ages, IQ scores of the two groups and the mean $\mathrm{ABC}$ scores of the ASP subjects. No differences in any of the thalamic volumes, the hemisphere volumes, TBV and volume fractions were observed between the two groups (Table 2). There were also no significant differences in thalamic volumes between the two groups after controlling for TBV ( $\mathrm{F}=0.004, \mathrm{p}=0.953)$.

The relationship between thalamic volumes and TBV was also examined in both groups. There was a strong significant correlation in the ASP group $(\mathrm{r}=0.75, \mathrm{p}<0.01)$ and a moderate significant correlation in the control group $(\mathrm{r}=0.51, \mathrm{p}=0.04)$.

No significant correlations were seen between thalamic volumes and age or IQ scores for both groups.

In addition, the ASP group showed a significantly greater laterality as measured by the absolute value of left-minus-right

Table 2. Thalamic, hemispheric volumes, volume factions and laterality in ASP and control groups

\begin{tabular}{|c|c|c|c|c|c|}
\hline & ASP & Control & & Mann-Whitney U & \\
\hline & $\mathrm{N}=15$ & $\mathrm{~N}=15$ & $\mathrm{df}=28$ & test & $\mathrm{p}$ \\
\hline & Mean \pm SD & Mean \pm SD & $\mathrm{t}$ & $\mathrm{z}$ & \\
\hline Right thalamus volume & $5.80 \pm 0.59$ & $5.79 \pm 0.63$ & 0.04 & - & 0.965 \\
\hline Left thalamus volume & $5.55 \pm 0.59$ & $5.68 \pm 0.57$ & -0.61 & - & 0.547 \\
\hline Total thalamus volume & $11.37 \pm 1.19$ & $11.48 \pm 1.18$ & -0.27 & - & 0.783 \\
\hline Right hemisphere volume & $598.83 \pm 83.00$ & $609.46 \pm 48.77$ & - & -0.560 & 0.595 \\
\hline Left hemisphere volume & $589.19 \pm 83.29$ & $599.25 \pm 50.72$ & - & -0.601 & 0.567 \\
\hline TBV & $1188.03 \pm 166.17$ & $1208.72 \pm 99.32$ & - & -0.560 & 0.595 \\
\hline Right volume fraction (\%) & $0.97 \pm 0.08$ & $0.95 \pm 0.09$ & 0.71 & - & 0.482 \\
\hline Left volume fraction (\%) & $0.94 \pm 0.07$ & $0.95 \pm 0.09$ & -0.09 & - & 0.925 \\
\hline Total volume fraction (\%) & $0.96 \pm 0.07$ & $0.95 \pm 0.09$ & 0.32 & - & 0.748 \\
\hline Thalamic Laterality & $-0.25 \pm 0.10$ & $-0.11 \pm 0.22$ & -2.22 & - & $0.034^{*}$ \\
\hline Laterality index (\%) & $-2.28 \pm 0.98$ & $-0.97 \pm 1.97$ & -2.30 & - & $0.029^{*}$ \\
\hline
\end{tabular}

All volumes represented in $\mathrm{mm}^{3}{ }^{*} \mathrm{p}<0.05$. ASP: Asperger's disorder group, SD: standard deviation, TBV: total brain volume 
thalamus difference as well as a significantly greater laterality index than the control group (Table 2). In the ASP group, there was a significant correlation between laterality index and $A B C$ language scores $(r=0.537, p=0.039)$ also between laterality and $\mathrm{ABC}$ language scores $(\mathrm{r}=0.541, \mathrm{p}=0.037)$.

No significant differences between the two groups were observed in right and left thalamic NAA, Cho and Cre concentrations (Table 3).

\section{DISCUSSION}

To the best of our knowledge, this is the first structural MRI study to report significantly increased laterality of the thalamus in children and adolescents with ASP. The ASP group also exhibited a significant relationship between laterality index and level of language abnormalities.

In the current study, the subjects with ASP showed no significant differences in thalamic volumes or TBV compared to healthy subjects. These results are consistent with most of the previous structural MRI studies examining the volume of the thalamus in autism or ASP. ${ }^{3-6,14}$ For example, the only previous study involving the subjects with ASP aged between 10-35 years old reported no volumetric difference with controls. ${ }^{5}$

A positive correlation between the thalamic volumes and TBV was observed in the ASP group as well as in the control group in the present study. This finding is consistent with a similar study that investigated thalamic volumes in children and adolescents with autism. Hardan et al. ${ }^{6}$ compared thalamic volumes of 18 autistic boys aged $8^{-} 15$ years old with normal controls and found no significant structural difference. However, our results are discordant with the results of other investigations reporting a significant correlation between thalamic volumes and TBV in controls but not in the autism or ASP group. ${ }^{3-5}$ The difference between the present study and several others $^{3-5}$ is most likely related to the difference in the age ranges of the subjects.

Table 3. Metabolite concentrations of right and left thalamus in ASP and control groups

\begin{tabular}{|c|c|c|c|c|}
\hline & \multirow{3}{*}{$\begin{array}{c}\text { ASP } \\
\mathrm{N}=15 \\
\text { Mean } \pm \text { SD }\end{array}$} & \multirow{3}{*}{$\begin{array}{c}\text { Control } \\
\mathrm{N}=15 \\
\text { Mean } \pm \text { SD }\end{array}$} & \multirow{2}{*}{\multicolumn{2}{|c|}{$\begin{array}{l}\mathrm{t} \text {-test } \\
\mathrm{df}=28\end{array}$}} \\
\hline & & & & \\
\hline & & & $\mathrm{t}$ & $\mathrm{p}$ \\
\hline Right NAA & $3.24 \pm 0.60$ & $3.21 \pm 0.76$ & 0.139 & 0.890 \\
\hline Right Cho & $1.85 \pm 0.66$ & $1.98 \pm 0.36$ & 0.608 & 0.548 \\
\hline Right Cre & $1.80 \pm 0.39$ & $1.74 \pm 0.45$ & 0.328 & 0.745 \\
\hline Left NAA & $3.30 \pm 0.53$ & $3.31 \pm 0.73$ & 0.026 & 0.979 \\
\hline Left Cho & $1.78 \pm 0.41$ & $1.90 \pm 0.43$ & 0.775 & 0.445 \\
\hline Left Cre & $1.82 \pm 0.54$ & $1.93 \pm 0.33$ & 0.624 & 0.538 \\
\hline
\end{tabular}

ASP: Asperger's disorder group, SD: standard deviation, NAA: NAcetylaspartate, Cre: Creatine, Cho: Choline
In the present study, a significantly greater left-minus-right thalamus difference was found as well as a significantly greater laterality index in the ASP group. Similarly, Tsatsanis et al. ${ }^{3}$ reported a significantly greater absolute left-minus-right thalamus difference in the autism group and concluded that "this outcome is consistent with the more general finding in individuals with autism of a lack of the expected scaling relationship in the thalamus relative to controls". However, our finding is inconsistent with the results of other previous studies, one of which found no differences in the laterality index in subjects with autism ${ }^{4}$ and the other, in subjects with ASP. ${ }^{5}$

Additionally, the current study provides evidence of a relationship between an abnormally increased laterality index in thalamus and clinical features, particularly the language abnormalities in boys with ASP. The involvement of the thalamus in language processing in healthy people has been established in many reports. Activation of the left but not right thalamus during linguistic tasks, such as word generation, semantic and phonemic fluency tasks has been identified in functional MRI studies. ${ }^{15}$ In a PET study, reduced activation was found in the left frontal region and left thalamus during verbal auditory and expressive language in the autistic group. This finding suggests that the serotonergic abnormalities in the dentato-thalamo-cortical pathway may lead to the receptive and expressive language deficits observed in autism. ${ }^{16}$ Although the current ICD and DSM manuals require normal early language development for a diagnosis of ASP to be made, the majority of children with ASP have some abnormalities in the development of spoken language such as articulation problems, pragmatic difficulties, abnormalities in prosody and also language comprehension problems. ${ }^{17}$ The findings of the current study of increased asymmetry in the thalamus and its relationship with language abnormalities in children and adolescents with ASP, supports the role of the thalamus in language abnormalities observed even in mild forms of autism, such as ASP.

In the present study, subjects with ASP showed no significant differences in thalamic metabolite concentrations compared to healthy subjects. Previous studies investigating the thalamic metabolite concentrations in autism have presented conflicting results. Our results are concordant with one study that examined the metabolite concentrations of thalamus in children and adolescents (age range $5-16$ years) with autism and reported no significant chemical abnormalities. ${ }^{18}$ Friedman et al. ${ }^{19}$ found significantly reduced NAA concentrations in the right, and reduced Cre and Cho concentrations in the left thalamus in a group of very young children with autism. In a study of 18 HFA children and adolescents aged mean 11.9 years old, using MRI and ${ }^{1} \mathrm{H}-\mathrm{MRS}$, while no difference was found in respect of thalamic volume between the autism group and the healthy controls, the NAA level measured in the left 
thalamus of the autism group was found significantly low. They also, observed a relationship between the measures of sensory abnormalities and thalamic metabolites. ${ }^{6}$ Additionally, reduced NAA level of autistic children is reported in a metaanalysis of ${ }^{1} \mathrm{H}$-MRS studies. ${ }^{20}$ Those inconsistencies in results across studies can be explained by the differences in sample characteristics (age, gender, IQ, comorbidity, medication) and methodological differences in ${ }^{1} \mathrm{H}$-MRS acquisition.

The findings of this study should be interpreted with caution due to the small sample size and the inclusion of males only. Second, a reliable diagnostic instrument for the diagnosis of ASP could not be used due to the unavailability of the Turkish versions of any diagnostic interviews for autism or ASP.

The homogenity of the sample (inclusion of subjects with ASP only) and the inclusion of the well matched groups in terms of age, gender, dominant hand side and IQ scores are the relative strengths of this study. The studies comparing thalamic volumes in autism mostly have sample groups with a very wide age range including children and adults. Therefore, the sample group of the current study was formed only of children and adolescents with ASP. This makes our study different from previous studies with the narrowing of the age range, and forming a group homogenous in terms of diagnosis.

In conclusion, it can be said that the findings from this investigation suggest that alterations from typical patterns of thalamus development are observed in children and adolescents with ASP. Longitudinal studies with larger sample sizes are needed to fully understand the developmental course and the role of the thalamus, especially in the area of language problems in autism.

\section{Acknowledgments}

This study was supported by Ondokuz Mayis University Scientific Research Funding.

\section{REFERENCES}

1. American Psychiatric Association. Diagnostic and Statistical Manual of Mental Disorders, Fourth Edition. Washington, DC: American Psychiatric Press; 2000.

2. Katz LC, Shatz CJ. Synaptic activity and the construction of cortical circuits. Science 1996;274:1133-1138.

3. Tsatsanis KD, Rourke BP, Klin A, Volkmar FR, Cicchetti D, Schultz RT. Reduced thalamic volume in high-functioning individuals with autism. Biol Psychiatry 2003;53:121-129.

4. Hardan AY, Girgis RR, Adams J, Gilbert AR, Keshavan MS, Minshew
NJ. Abnormal brain size effect on the thalamus in autism. Psychiatry Res 2006;147:145-151.

5. Hardan AY, Girgis RR, Adams J, Gilbert AR, Melhem NM, Keshavan MS, et al. Brief report: abnormal association between the thalamus and brain size in Asperger's disorder. J Autism Dev Disord 2008;38: 390-394.

6. Hardan AY, Minshew NJ, Melhem NM, Srihari S, Jo B, Bansal R, et al. An MRI and proton spectroscopy study of the thalamus in children with autism. Psychiatry Res 2008;163:97-105.

7. Tamura R, Kitamura H, Endo T, Hasegawa N, Someya T. Reduced thalamic volume observed across different subgroups of autism spectrum disorders. Psychiatry Res 2010;184:186-188.

8. Perich-Alsina J, Aduna de Paz M, Valls A, Muñoz-Yunta JA. Thalamic spectroscopy using magnetic resonance in autism. Rev Neurol 2002;34 (Suppl 1): S68-S71.

9. Yilmaz-Irmak T, Tekinsav-Sutcu WS, Aydin A, Sorias O. An investigation of validity and reliability of Autism Behavior Checklist (ABC). Turk J Child Adolesc Ment Health 2007;14:13-23.

10. Kaufman J, Birmaher B, Brent D, Rao U, Flynn C, Moreci P, et al. Schedule for affective disorders and schizophrenia for school-age children-present and lifetime version (K-SADS-PL): initial reliability and validity data. J Am Acad Child Adolesc Psychiatry 1997;36:980-988.

11. Gokler B, Unal F, Pehlivanturk B, Cengel Kultur E, Akdemir D, Taner Y. Reliability and validity of schedule for affective disorders and schizophrenia for school age children-present and lifetime version-turkish version (K-SADS-PL-T). Turk J Child Adolesc Ment Health 2004;11: 109-116.

12. Sahin B, Ergur H. Assessment of the optimum section thickness for the estimation of liver volume using magnetic resonance images: a stereological gold standard study. Eur J Radiol 2006;57:96-101.

13. Sahin B, Elfaki A. Estimation of the volume and volume fraction of brain and brain structures on radiological images. Neuroquantology 2012; 10:87-97.

14. Haznedar MM, Buchsbaum MS, Hazlett EA, LiCalzi EM, Cartwright C, Hollander E. Volumetric analysis and three-dimensional glucose metabolic mapping of the striatum and thalamus in patients with autism spectrum disorders. Am J Psychiatry 2006;163:1252-1263.

15. Hebb AO, Ojeman GA. The thalamus and language revisited. Brain Lang 2013;126:99-108.

16. Müller RA, Chugani DC, Behen ME, Rothermel RD, Muzik O, Chakraborty PK, et al. Impairment of dentato-thalamo-cortical pathway in autistic men: language activation data from positron emission tomography. Neurosci Lett 1998;245:1-4.

17. Gillberg CA. Guide to Asperger Syndrome. Cambridge: Cambridge University Press; 2002.

18. Levitt JG, O’Neill J, Blanton RE, Smalley S, Fadale D, McCracken JT, et al. Proton magnetic resonance spectroscopic imaging of the brain in childhood autism. Biol Psychiatry 2003;54:1355-1366.

19. Friedman SD, Shaw DW, Artru AA, Richards TL, Gardner J, Dawson $\mathrm{G}$, et al. Regional brain chemical alterations in young children with autism spectrum disorder. Neurology 2003;60:100-107.

20. Aoki Y, Kasai K, Yamasue H. Age-related change in brain metabolite abnormalities in autism: a meta-analysis of proton magnetic resonance spectroscopy studies. Transl Psychiatry 2012;2:e69. 